\title{
Interplay of multivalency and optical properties of quantum dots: Implications for sensing and actuation in living cells
}

\section{Guillermo Menéndez ${ }^{\mathrm{a}}$, María Julia Robertia ${ }^{\mathrm{a}}$ Valeria Sigot $^{\mathrm{b} \zeta}$, María Etchehon ${ }^{\mathrm{a}}$, Thomas M. Jovin ${ }^{b}$, and Elizabeth A. Jares-Erijman ${ }^{a^{*}}$}

\author{
${ }^{\text {a}}$ Departamento de Química Orgánica, Facultad de Ciencias Exactas y Naturales, Universidad de \\ Buenos Aires (UBA), 1428 Buenos Aires, Argentina; \\ ${ }^{\mathrm{b}}$ Laboratory of Cellular Dynamics, Max Planck Institute for Biophysical Chemistry, 37077 \\ Göttingen, Germany; Laboratorio Max Planck de Dinámica Celular, FCEyN, UBA, Buenos Aires
}

\begin{abstract}
Quantum dots (QDs) are unique probes due to their special properties (brightness, photostability, narrowband emission and broadband absorption), and excellent bio(chemical)compatibility for imaging structures and functions of living cells. When functionalized with ligands, they enable the recognition of specific targets and the tracking of dynamic processes for extended periods of time, detecting biomolecules with a sensitivity extending to the single molecule level. Thus, devices and probes based on such nanoparticles are very powerful tools for studying essential processes underlying the functions and regulation of living cells.

Here we present nanosensors and nanoactuators based on QDs in which the multivalency of these particles plays an essential role in the functionality and sensing characteristics of the nanodevices. Two examples are discussed, the first being $\mathrm{pH}$ nanosensors based on the interplay of the multivalency and energy transfer between the nanoparticles and small molecules on their surface, and the second nanoactuators in which a controlled number of molecules of the amyloid protein $\alpha$-synuclein (AS) specifically regulate the aggregation of fluorescently labeled bulk AS protein both in vitro and in live cells.
\end{abstract}

Keywords: $\quad$ pH nanosensors, nanoeffectors, amyloid protein aggregation, $\alpha$-synuclein, Parkinson's disease, imaging, confocal microscopy.

\section{INTRODUCTION}

Quantum dots (QDs) are exceptional probes due to their unique properties (brightness, photostability, narrowband emission and broadband absorption), and capability of broad spectrum bioconjugation required for the imaging of structures and functions in living cells. Complexed to ligands, QDs can recognize specific targets and track them in the course of dynamic processes for extended periods of time [1-9]. Due to their facile detection as individual particles, QDs offer a sensitivity of detection of biomolecules extending to the single molecule level. Thus, one can study the essential processes underlying the functions and regulation of living cells with probes providing the selectivity required for dissecting complex networks .

Characteristics related to the local density and nature of molecular components on the surface of QDs can be exploited in cells and tissues for purposes other than mere detection. The systematic "molecular engineering" of the surface composition has been a central feature in numerous applications ranging beyond biosensing to cell delivery and release and to the activation of individual reactions and entire pathways, as featured in the last section of this report.

${ }^{\zeta}$ present address: Laboratorio de Investigaciones en Criobiología (LIC), Facultad de Ciencias Bioquímicas y Farmacéuticas, Universidad Nacional de Rosario, S2002LRK Rosario, Santa Fe, Argentina.

* corresponding author: eli@qo.fcen.uba.ar, phone: +54-114-5763448, fax: ++54-114-5763346

Colloidal Quantum Dots for Biomedical Applications IV, edited by Marek Osinski, Thomas M. Jovin, Kenji Yamamoto, Proc. of SPIE Vol. 7189, 71890P - @ 2009 SPIE · CCC code: 1605-7422/09/\$18 - doi: 10.1117/12.816896 
An essential requirement for the rational design of sensors, effectors and other devices is an understanding of how nanomaterials interact with intracellular structures and processes. The level of complexity reflects structural and dynamic features necessarily operating in a confined situation. Multivalency is an aspect that has to be considered appropriately, inasmuch as its interplay with the photophysical properties of QDs can lead to unique patterns of sensing and initiation of activity.

A widespread tendency is to consider the existence of multiple groups on the surface of NPs as disadvantageous in comparison with single attachment or reactive points, the rationale being that extensive conjugation may lead to a distribution of NP subpopulations differing in particle-label stoichiometries, to undesirable and nonphysiological crosslinking, and other effects. Procedures for achieving precise control of the number of sites (down to single moieties) have been developed [10]. However, under certain circumstances the presence of a large number of sites may offer distinct advantages. In fact, numerous studies demonstrate that multiple identical or different molecules on the surface of NPs can be exploited for a wide variety of purposes, ranging from the control of optical or physical properties to the concurrent regulation of different functions.

Commercially available NPs coated with a polymeric layer to isolate the core-shell structure from the aqueous environment have diameters of $15-20 \mathrm{~nm}$ and may extend to twice these values if covered with PEG. Is this considerable size important such that QDs can be considered to operate more as platforms with a high surface area and extensive multivalency rather than as single molecular entities? The answer is probably affirmative, in that size not only influences photophysical properties such as quantum confinement but also determines the number of molecules that can be attached to the nanoparticle as well as their arrangement on the surface.

QDs have proven to be very useful as FRET donors and acceptors in numerous applications [11,12]. As donors, QDs often are attached to multiple acceptors, thereby achieving efficient quenching of the emission. There have been relatively few reports of experiments based on imaging [1,3, 4, 6, 13-17]. In our own studies of cellular signaling mediated by growth factors [5, 9, 18-19] and their receptors, QDs conjugated with ligands have revealed the many features of binding, endocytosis and intracellular trafficking. In one instance, a biotinylated organic dye FRET acceptor served to demonstrate the existence of a novel mechanism for the retrograde transport of activated EGF receptor (EGFR) on filopodia, cellular extensions with a core of actin filaments [5]. Figure 1 depicts the strategy for using QDs attached to ligands as vehicles for specific targeting of cell surface receptors. In this case, QD-bound biotinylated EGF bound efficiently to the EGFR and through this interaction, provided insight into the early stages of internalization of this prototypic family of receptor tyrosine kinases (RTKs).
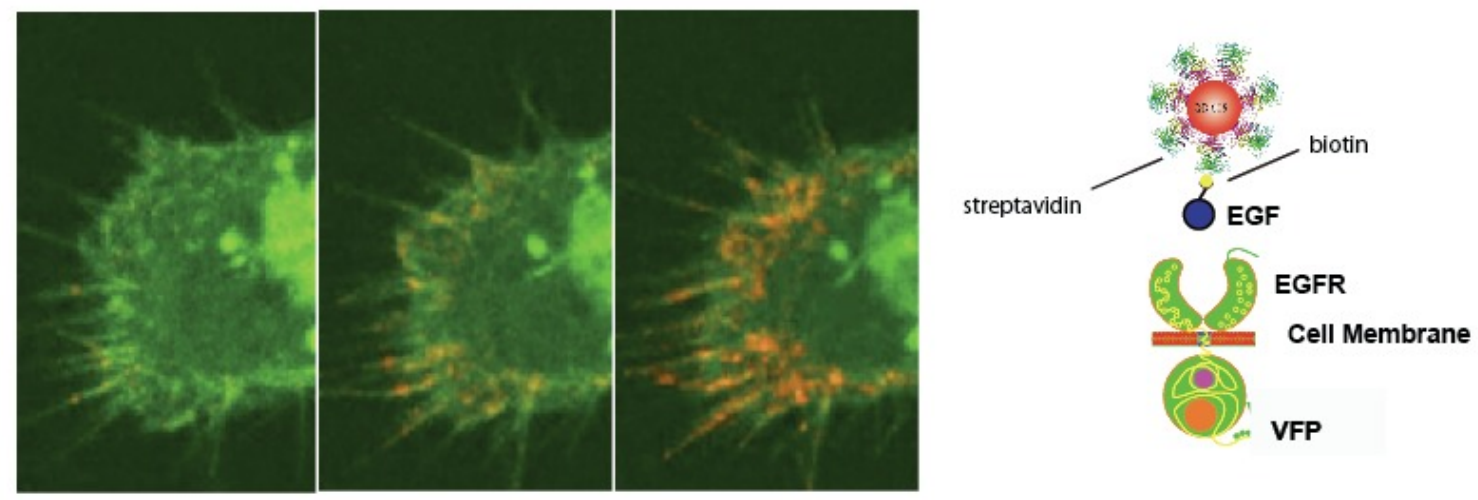

Figure 1. Strategy for studying the binding of QD-EGF to the EGF receptor (EGFR) and the resultant activation and endocytosis of the receptor into living cells. The image displays A431 cells expressing ERB1-EGFP 20 min after addition of biotinylated EGF bound to Streptavidin QD-605. For further details see [9]. 
QD donors have been applied extensively in FRET-based assays of enzymatic activities, with the systematic introduction of multifunctionality being a major issue ([20] and references therein). The distance dependence of FRET operating via surface-bound or nearby small-molecule acceptors reflects the complex interplay between factors such as stoichiometry, spatial distribution and orientation, composition (passivation shell, capping moieties), and shape. (QDs emitting at $>600 \mathrm{~nm}$ tend to be non-spherical and demonstrate finite emission polarization, [21]).

Optical properties such as fluorescence intensity or lifetime can also be tailored with FRET acceptors. The design of FRET based biosensors for monitoring defined states generally requires control of the number of acceptors associated with each QD donor [22]. These features are exemplified in the following section.

\section{RESULTS}

\section{$2.1 \mathrm{pH}$ sensors based on commercially available QD donors and multiple pH sensitive small molecule acceptors} Reversible $\mathrm{pH}$ sensors based on QD donors and $\mathrm{pH}$ sensitive dyes as acceptors have been recently reported [24]. These sensors use a squaraine dye with pKa 8.5 covalenty linked to the nanoparticles, and are evaluated by a ratiometric change in fluorescence intensity.

In our own development of QD-based $\mathrm{pH}$ sensors, we sought a system capable of operating over a wide $\mathrm{pH}$ range, including the physiological cytoplasmic milieu as well as the acidic intracellular compartments characteristic of endocytic transport and processing. We defined further requirements: (i) a sensor based on commercially available nanoparticles and $\mathrm{pH}$ indicators; and (ii) readout both by steady-state and time resolved spectroscopy (fluorescence lifetimes), which in the latter case is largely independent of probe and target concentration and well suited for imaging systems.

The above considerations led us to the choice of Streptavidin-conjugated-ITK-QD605 (Invitrogen) donors and biotinylated carboxynaphthofluorescein (bCNF) acceptors (Fig. 2a).

The choice of a non-covalent $\mathrm{pH}$ indicator ligand provides for the systematic selection of donor/acceptor ratios (and thereby dynamic range) and the concurrent conjugation to other biotinylated molecules, such as a growth factor like EGF (Fig. 1) for specific targeting of the $\mathrm{pH}$ sensor. The absorption spectra of the acidic and basic forms of the CNF indicator (pK 7.65; [24]), compared to the emission spectrum of the QD605, are shown in Fig. 2b. Only the basic form of the indicator absorbs well in the emission band of the QD and thus FRET would be expected to operate much more efficiently at high $\mathrm{pH}$.

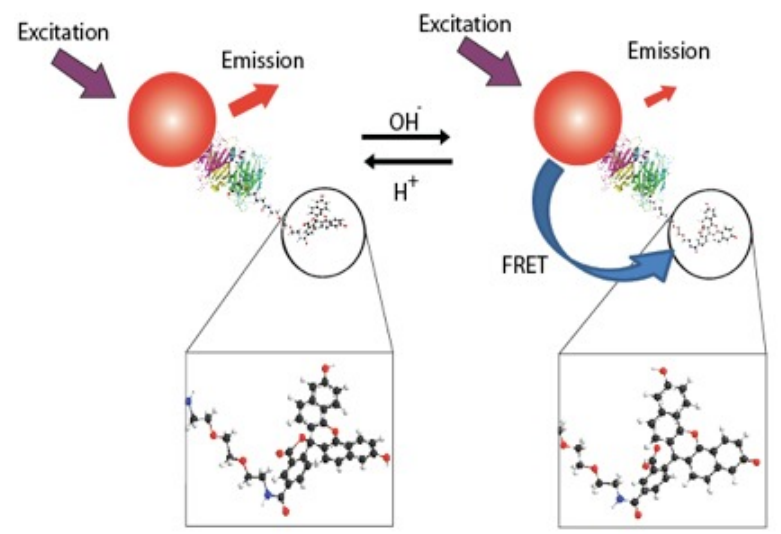

b

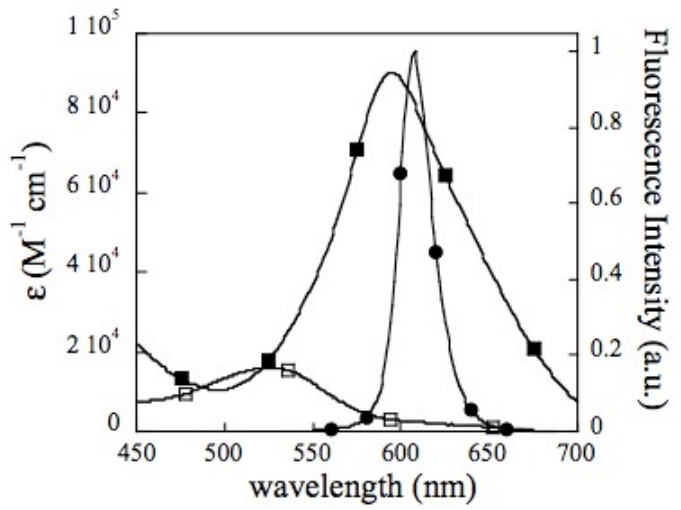

Fig. 2 . Conceptual design of streptavidin-QD605-bCNF pH sensor. a) schematic representation; b) QD emission spectrum (•) and the two absorption spectra (acidic, $\square$; basic; $\square$ ) of CNF.

The titration of Streptavidin-ITK-QD605 at $\mathrm{pH} 10$ with bCNF led to significant quenching of the QD donor, achieving $80 \%$ at approximately 70 equivalents of dye (Fig. 3a). As expected, the emission of QDs loaded with 30 equivalents of bCNF increased (due to dequenching) as the $\mathrm{pH}$ was reduced progressively to $\mathrm{pH} 4$. The two 
fluorescence lifetimes also increased accordingly, yielding an amplitude-weighted mean value $\left(\tau_{\text {mean }}\right)$ between 4 and 9 ns (Fig. 3b). A remarkable result was the linear dependence of $\tau_{\text {mean }}$ on $\mathrm{pH}$ over the wide range of 5.5-9.5, obeying the relationship $\mathrm{pH}=13.4-0.92 \tau_{\text {mean }}$ (ns).

a

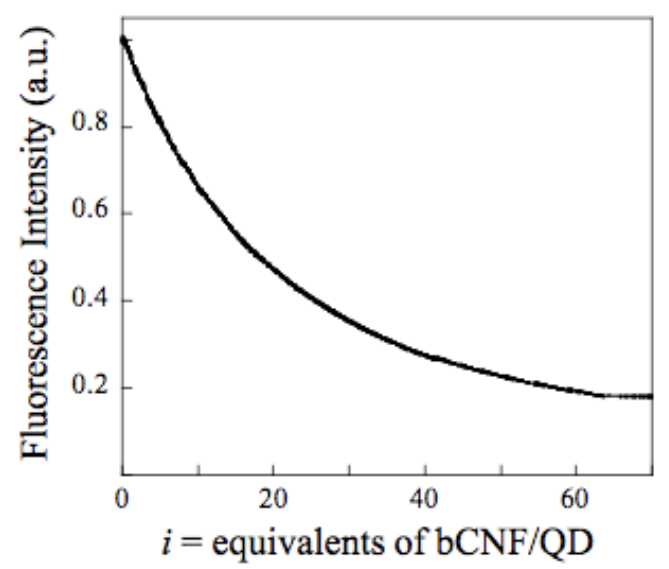

b

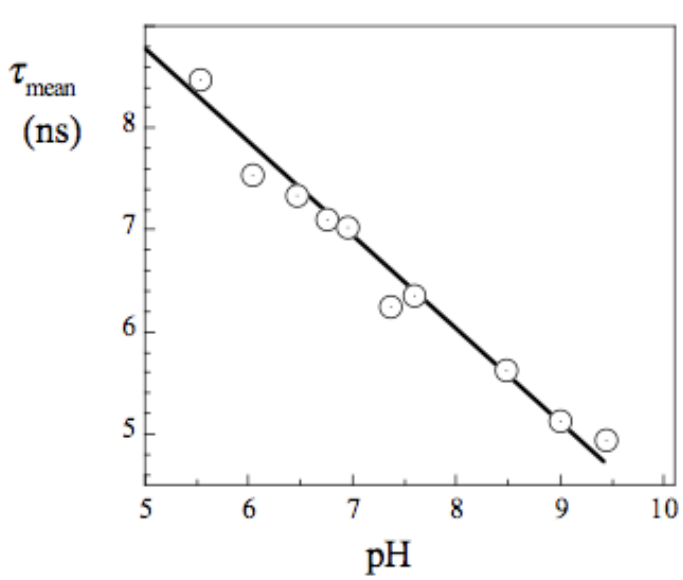

Figure 3. Dependence of steady-state fluorescence on loading (a) and mean fluorescence lifetime $\left(\tau_{\text {mean }}\right)$ on $\mathbf{p H}(\mathbf{b})$. a) $\mathrm{pH} 10$. The control (upper trace) was performed using QD saturated with free biotin prior to the titration. b) $i=30 \mathrm{bCNF} / \mathrm{QD}$. The fluorescence lifetimes were measured in an Olympus inverted IX71 microscope to which was attached an LED-based (373 nm) IBH (now Horiba Jobin Yvon) TCSPC FluoroCube system.

Another interesting feature of the two fluorescence lifetimes was an increase with $\mathrm{pH}$ of the relative amplitude of the short component. Numerous potential photophysical models for the system were explored in order to account quantitatively for the lifetime data, the dependence of the steady-state QD fluorescence on $\mathrm{pH}$ and loading ( $i$ probes/QD), and an apparent quenching of the bCNF acceptor. A detailed discussion of these issues is outside the scope of this report, yet one potential scheme is featured in Figure 4. Two interesting assumptions involve (i) two interconverting excited states of the QD, differing in their geometric distribution and thus transfer properties, and the reverse transfer of CNF to the QD due to the finite overlap of the CNF emission with the QD absorption. In view of the two forms of the CNF populated according to the $\mathrm{pH}$, four evaluations of FRET overlap integrals [25] are required.
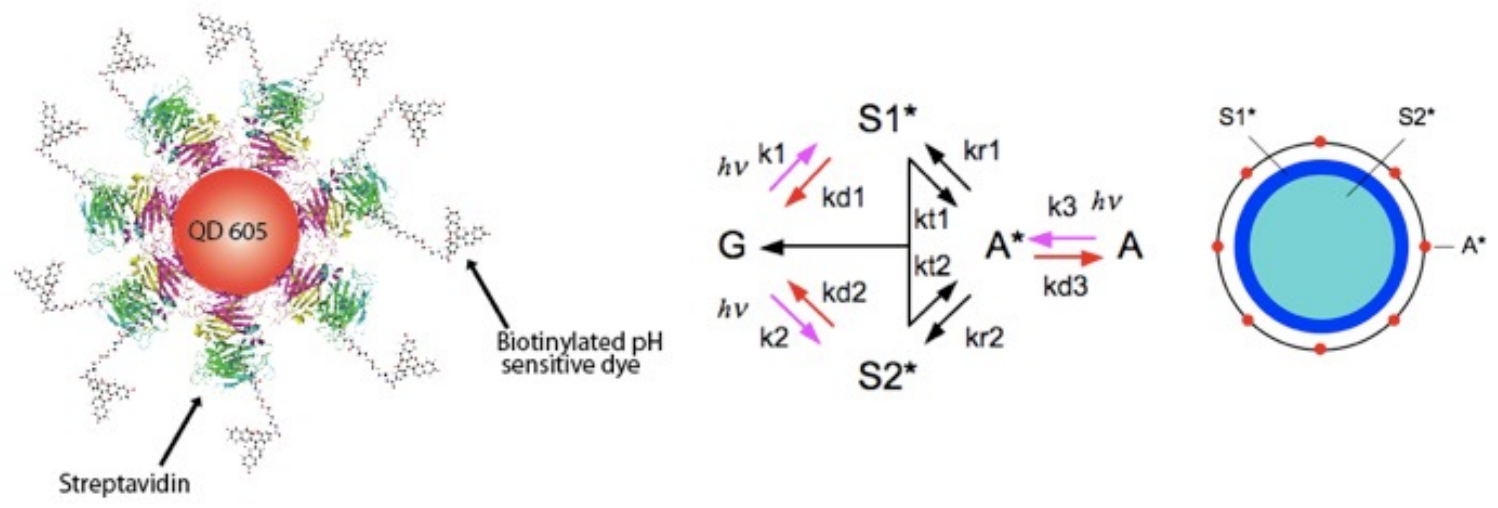

Figure 4. Rationale and a photophysical scheme for QD-CNF pH sensor. Left, multiple bCNF acceptors on the surface of Streptavidin-ITK-QD605. Right, photophysical model of the system considering both forward (QD (D) $\rightarrow$ CNF (A) and reverse $(\mathrm{CNF} \rightarrow \mathrm{QD})$ FRET, direct excitation of both QD and acceptor, and two virtual excited states of the QD ("peripheral", S1*, and "core", S2*); the multiple acceptors are depicted as a shell surrounding the QD. 
a

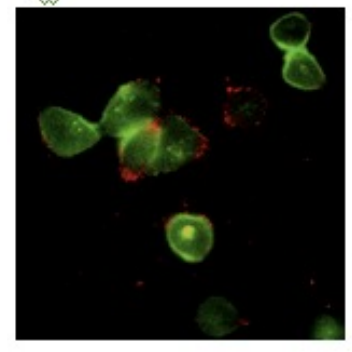

b

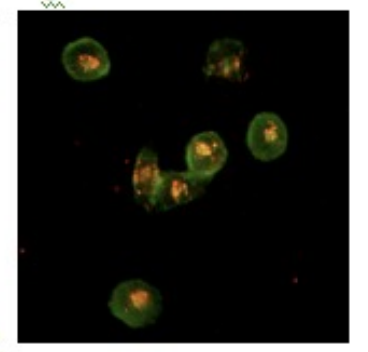

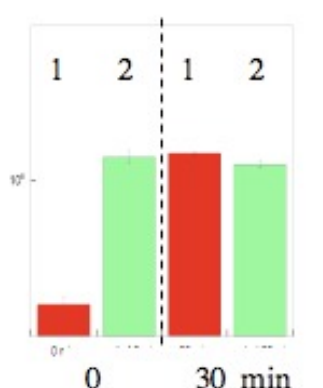

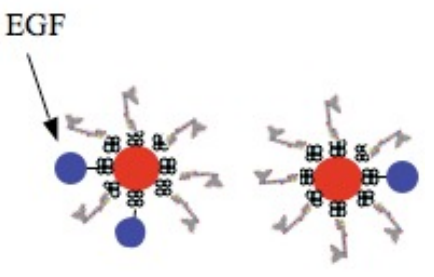

$30 \mathrm{~min}$

Figure 5. Live cell determinations of $\mathbf{p H}$ with QD nanosensors. Four equivalents of biotin-EGF and a saturating concentration of bCNF were added in sequence to Streptavidin-ITK-QD605. HeLa Cells were incubated with the nanoparticles (a, initial determination, $\mathrm{t}=0 ; \mathbf{b}, \mathrm{t}=30 \mathrm{~min}$ ). Intensities are shown at $\mathrm{t}=0$ and $\mathrm{t}=30 \mathrm{~min}$ for nanosensors (1) and QD controls (2). Lifetime at $\mathrm{t}=0 \mathrm{~min}$, corresponded to $\mathrm{pH} 7.2$ (a) and at $\mathrm{t}=30 \mathrm{~min}$, to $\mathrm{pH} 7.2(\mathbf{b})$.

Figure 5 shows imaging with the QD nanosensors in living HeLa cells. Lifetime determinations were carried out on the signal arising from the complete field selected with a 605 bp emission filter to discriminate for the QD emission. During 30 min of incubation internalization occurred and was accompanied by an increase of the intensity of emission arising from the nanosensors. The $\mathrm{pH}$ changes derived from the determination using the calibration curve of Figure 3 demonstrated a change from $\mathrm{pH} 7.3$ to 7.2 [26].

\subsection{QDs nanoactuators in amyloid protein aggregation}

Engineering the surface of NPs can also be applied for the initiation and control of the self-assembly of biomolecules. In the case of the amyloid-forming proteins involved in most neurodegenerative diseases, such tools can provide important insights on the mechanism of fibrillation in living cells and organisms and also have great potential for very sensitive readouts in drug discovery efforts. A non-specific approach that takes advantage of the large surface area offered by most nanostructures was introduced recently [27]. Co-polymer particles, cerium oxide particles, QDs, and nanotubes were tested as nucleation agents for the aggregation of the protein human $\beta 2$-microglobulin. In the fibrillation process, nucleation is a (the?) key rate-determining step. The authors reported that the acceleration of nucleation depended on the extent and nature of the particle surface. The disadvantage of this procedure is that it lacks specificity, that is, it cannot discriminate between different molecules with the tendency to form aggregates, or molecules exhibiting affinities for such structures. In addition, aggregation under such conditions requires high concentrations of the nanostructures (100 nM in the case of QDs). Such concentrations can seriously perturb the biological systems under study.

We have developed an alternative strategy that offers very high specificity and at the time can be applied with very low concentration of QDs [28]. By using QDs bearing a controlled number of molecules of $\alpha$-synuclein (AS) molecules, a protein related to the pathogenicity of Parkinson's disease, it was possible to combine the specific detection of aggregation in living cells, with a specific enhancement of the nucleation process triggering fibrillation. Saturation of the QDs with AS units, led to the formation of "hot spots" with high nucleation potential, as described below.

Great efforts are devoted to revealing the mechanisms underlying the physiological function(s) of AS and the conditions leading to its pathological self-association [29]. In living cells and organisms, oxidative stress conditions can be created by exposure to a variety of chemical agents such as $\mathrm{FeCl}_{2}$ or $\mathrm{H}_{2} \mathrm{O}_{2}$. These compounds initiate a complex cascade of events leading to different disfunctions, including that of amyloid protein aggregation. In the case of the ASmodified QDs acting as specific nucleation sites, AS aggregation was initiated by a physical-chemical process inherent to the NP rather than a consequence of complex mechanisms involving a variety of molecular entities. However, external stimulation, such as by oxidative stress, was required in the case of another expression probe we devised [30] for AS, an AS mutant (AS-TC) involving a fusion of a 12-aa tag with the tetracysteine motif that specifically binds fluorogenic biarsenical compounds (e.g. FlAsH, ReAsH). The functional properties of wild type AS protein were retained, such that aggregates, e.g. induced by exposure of cells to $\mathrm{Fe}^{++}$, could be readily detected and characterized both in vitro and in live cells [30]. Figure 6 depicts the function of AS-QDs in vitro. The aggregation kinetics were monitored in the presence and in the absence of the NPs. The association of QDs with nascent fibers was confirmed by 
AFM (upper panel), and the acceleration of aggregation (shorter lag phase) was demonstrated by the standard Thioflavin T (ThioT) assay.
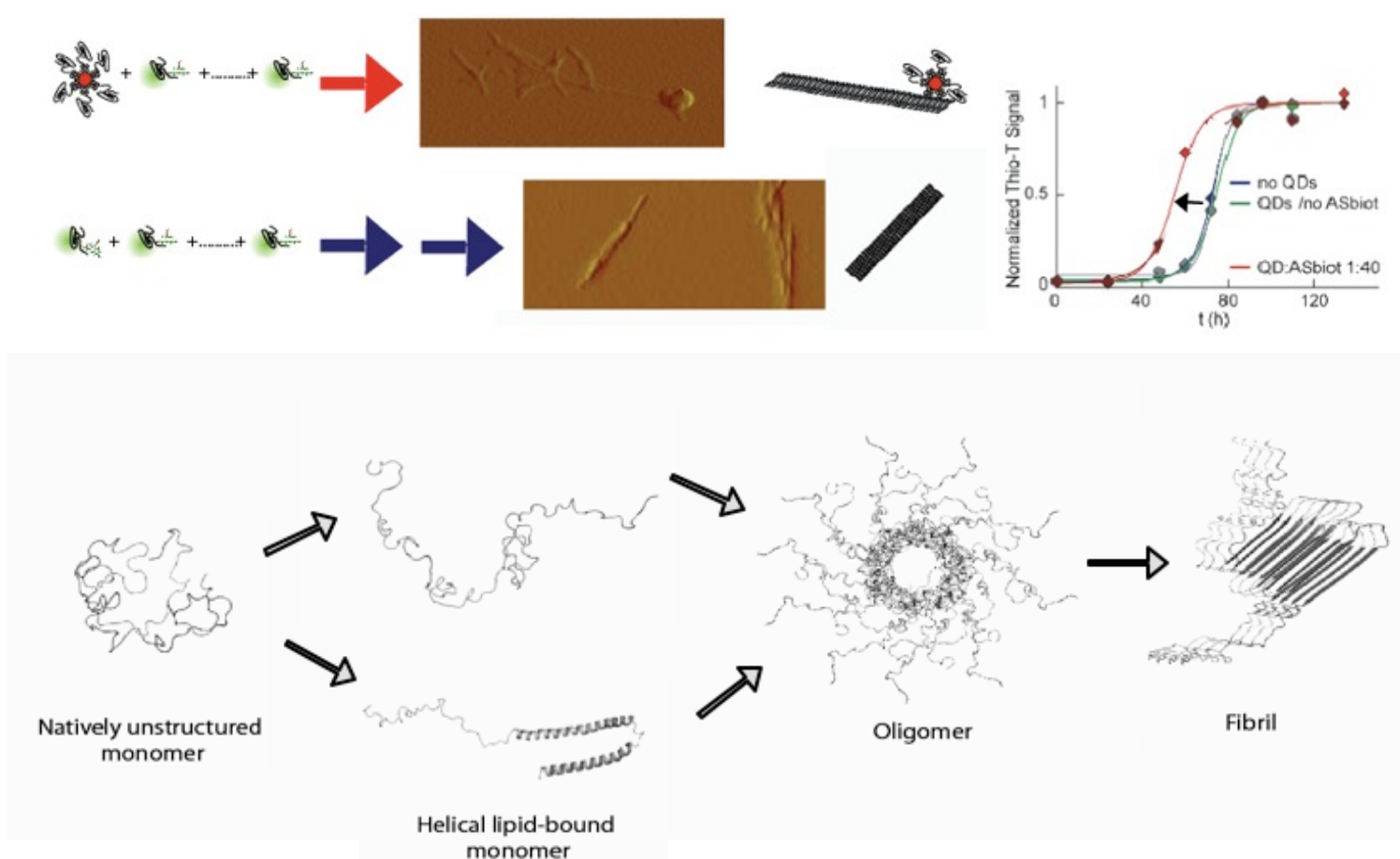

Figure 6. QDs acting as specific nucleation sites for $\boldsymbol{\alpha}$-synuclein (AS) in vitro. The aggregation of AS was monitored by ThioT fluorescence and by AFM. $5 \mathrm{nM}$ (40:1 protein:QD ratio) AS-QDs605 incubated together with $100 \mathrm{mM}$ of AS displayed a shorter lag and faster aggregation than in absence of QDs. The upper arrow indicates the aggregation course leading to fibrils attached to NPs, as confirmed by AFM (upper panel). The lower arrows show fibril morphologies after aggregation in the absence of the QD nucleation centers. Lower panel: aggregation of AS from a natively unstructured protein to oligomers and fibrils.

The combination of this construct with the QD reagents described above was particularly useful, as demonstrated in studies of living cells (HeLa, Figure 7). Varying (0-20 nM) concentrations of AS-QDs were microinjected together with $100 \mathrm{mM}$ of AS-TC. In the presence of $2 \mathrm{nM}$ QDs (Figure 6, upper panel), cells displayed a markedly increased percentage of aggregates, observed $24 \mathrm{~h}$ after microinjection, compared to the controls (lower panel) lacking QDs. In this strategy, the QDs functioned in a dual manner, i.e. both as biomarkers and "nanoactuators," i.e. specific nucleation agents. As biomarkers at very low concentrations they allowed the facile detection of AS molecules in fibrils, despite the presence of a vast excess of monomeric protein. The dual labeling with the biarsenical compounds provided a further "photophysical" enhancement of sensitivity [28]. These techniques will also benefit from improved biarsenicals that we have synthesized as an associated activity of the above investigations [31]. In addition, we anticipate that dual function NPs will undoubtedly be exploited in numerous other systems. 


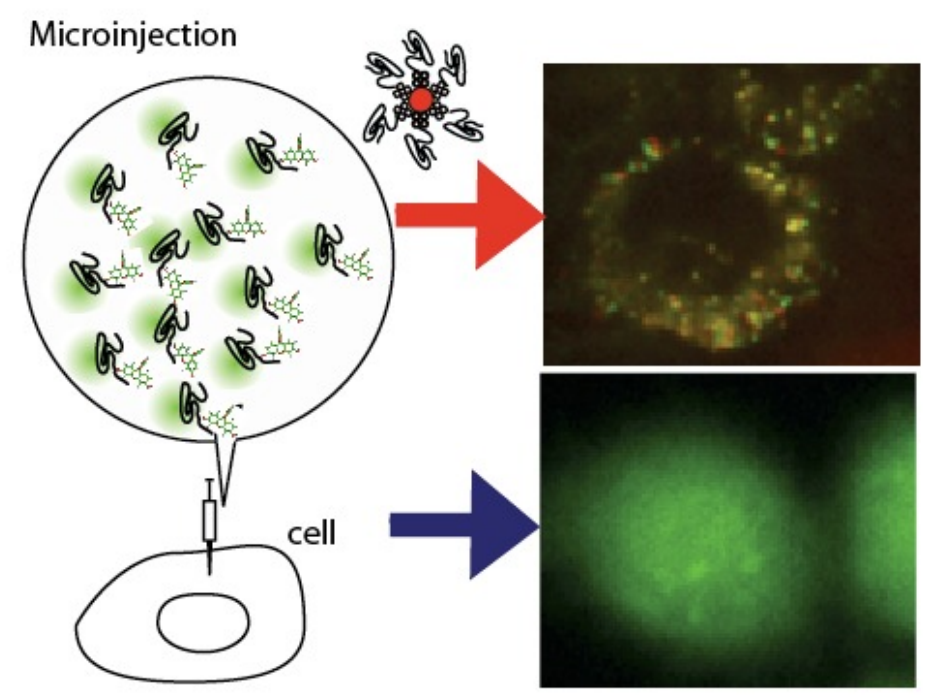

Figure 7. QDs acting as specific nucleation sites for $\alpha$-synuclein (AS) in living cells. Cells were microinjected with AS in the presence or absence of AS-QDs-605 (40:1 protein:QD) and observed after $24 \mathrm{~h}$ of incubation. A mixture $2 \mathrm{nM}$ AS-QDs-605 and 100 $\mathrm{mM}$ of AS-TC labeled with FlAsH was microinjected into HeLa cells (upper panel). QDs were detected, colocalizing to a pronounced degree with AS aggregates. Lower panel, microinjection of $100 \mathrm{mM}$ of AS-TC labeled with FlAsH in the absence of QDs.

In this report we have emphasized the increasing potential offered by the multivalency derived from introducing multiple sites on QDs. Such reagents offer unique opportunities for combining different functions such as sensing, targeting, cell penetration and delivery. In addition, by taking advantage of the particular chemistry at the surface of NPs, "smart" actuators can de devised and employed. The further combination with programmable inorganic and organic materials that demonstrate properties responsive to manipulation of temperature, light, electric and magnetic fields, $\mathrm{pH}$, ionic strength will enable a still greater increase in design complexity and utility. A golden age of smart nanodevices and nanomachines is in sight.

\section{ACKNOWLEDGMENTS}

E.A.J.-E. is funded by the Max Planck Society (Partner Group grant), ANPCyT, CONICET and UBACyT. This work was supported by the DFG Center for Molecular Physiology of the Brain (DFG CMPB), Cluster of Excellence 171 of the CMPB, in Göttingen, Germany, and the Max Planck Society (Toxic Protein Conformation project).

\section{REFERENCES}

[1] Cambi, A., Lidke, D., Arndt-Jovin, D., Figdor, C. and T. M. Jovin, "Ligand-conjugated quantum dots monitor antigen uptake and processing by dendritic cells" Nano Lett., vol. 7, 970-977 (2007).

[2] Fountaine, T.J., Wincovitch, S.M., Geho, D.H., Garfield, S.H. and Pittaluga, S., "Multispectral imaging of clinically relevant cellular targets in tonsil and lymphoid tissue using semiconductor quantum dots" Mod. Pathol., vol. 19, No. 9, 1181-1191 (2006).

[3] Li, Z.B., Cai, W. and Chen, X., "Semiconductor quantum dots for in vivo imaging" J. Nanosci. \& Nanotech., vol. 7, No. 8, 2567-2581 (2007).

[4] D. S. Lidke, and D. J. Arndt-Jovin, "Imaging takes a quantum leap" Rev. Med. Physiol., vol. 19, 2004, $322-325$ (2004). 
Lidke, D.S., Lidke, K.A., Rieger, B., Jovin, T.M. and Arndt-Jovin, D.J., "Reaching out for signals: filopodia sense EGF and respond by directed retrograde transport of activated receptors" J. Cell Biol., vol. 170, 619-626 (2005).

[6] Rhyner, M.N., Smith, A.M., Gao, X., Mao, H., Yang, L. and Nie S., "Quantum dots and multifunctional nanoparticles: new contrast agents for tumor imaging" Nanomed., vol. 1, No. 2, 209-217 (2006). Pinaud, F., Michalet, X., Bentolila, L.A., Tsay, J.M., Doose, S., Li, J.J., Iyer, G. and Weiss, S., "Advances in fluorescence imaging with quantum dot bio-probes" Biomater., vol. 27, No. 9, 1679-87 (2006).

[8] Gao, X., Cui, Y., Levenson, R.M., Chung, L.W. and Nie, S., "In vivo cancer targeting and imaging with semiconductor quantum dots" Nat. Biotechnol., vol. 22, No. 8, 969-76 (2004). Lidke, D.S., Nagy, P., Heintzmann, R., Arndt-Jovin, D.J., Post, J.N., Grecco, H.E., Jares-Erijman, E.A. and Jovin, T.M., "Quantum dot ligands provide new insights into erbB/HER receptor-mediated signal transduction" Nat. Biotechnol., vol. 22, No. 2, 198-203 (2004).

[10] Sperling, R. A., Pellegrino, T., Li, J.K., Chang W.A. and Parak W.J., "Electrophoretic separation of nanoparticles with a discrete number of functional groups" Adv. Func. Mater., vol. 16, 943-948 (2006).

Sapsford, K.E., Berti, L. and Medintz, I.L., "Materials for fluorescence resonance energy transfer analysis: Beyond traditional donor-acceptor combinations" Angew. Chem.-Int. Ed., vol. 45, No. 28, 4562-4588 (2006). Sapsford, K.E., Bradburneb, C., Delehantyb, J.B., and Medintz, I.L, "Sensors for detecting biological agents" Mater. Today, vol. 11, No. 3, 38-49 (2008).

Medintz, I.L., Uyeda, H.T., Goldman, E.R., and Mattoussi, H., "Quantum dot bioconjugates for imaging, labelling and sensing" Nat. Mater., vol. 4, No. 6, 435-446 (2005).

Lidke, K.A., Rieger, B., Lidke, D.S., Jovin, T.M, "The role of photon statistics in fluorescence anisotropy imaging" IEEE Trans. Image Process., vol. 14, No. 9, 1237-1245 (2005).

Jaiswal, J.K., Mattoussi, H., Mauro, J.M., Simon, S. M., "Long-term multiple color imaging of live cells using quantum dot bioconjugates" Nat. Biotechnol., vol. 21, 47-51 (2003).

Jaiswal, J.K., Goldman, E.R., Mattoussi, H. and Simon, S.M., "Use of quantum dots for live cell imaging" Nat. Methods, vol. 1, No. 1, 73-78 (2004).

Inoue, Y., Izawa, K., Yoshikawa, K., Yamada, H., Tojo and A., Ohtomo, K., "In vivo fluorescence imaging of the reticuloendothelial system using quantum dots in combination with bioluminescent tumour monitoring"

Eur. J. Nucl. Med. \& Mol. Imag., vol. 34, No. 12, 2048-2056 (2007).

Echarte, M.M., Bruno, L., Arndt-Jovin, D.J., Jovin, T.M. and Pietrasanta, L.I., "Quantitative single particle tracking of NGF-receptor complexes: transport is bidirectional but biased by longer retrograde run lengths" FEBS Lett., vol. 581, No. 16, 2905-2913 (2007).

Lidke, D.S., Nagy, P., Barisas, B.G., Heintzmann, R., Post, J.N., Lidke, K.A., Clayton, A.H., Arndt-Jovin, D.J. and Jovin, T.M., "Imaging molecular interactions in cells by dynamic and static fluorescence anisotropy (rFLIM and emFRET)" Biochem. Soc. Trans., vol. 31 1020-1027 (2003).

Susumu, K, Uyeda, H.T., Medintz, I.L., Pons, T., Delehanty, J.B., and Mattoussi, H., "Enhancing the stability and biological functionalities of quantum dots via compact multifunctional ligands" J. Am. Chem. Soc, vol. 129, 13987-13996 (2007).

Grecco, H.E., Lidke, K.A., Heintzmann, R., Lidke, D.S., Spagnuolo, C., Martinez, O.E., Jares-Erijman, E.A., and Jovin, T.M, "Ensemble and single particle photophysical properties (two-photon excitation, anisotropy, FRET, lifetime, spectral conversion) of commercial quantum dots in solution and in live cells" Micros. Res. Tech., vol. 65, No. 4-5, 169-179 (2005).

Medintz I. L., Konnert J. H., Clapp A. R., Stanish I., Twigg M. E., Mattoussi H., Mauro J. M. and Deschamps J. R, "A fluorescence resonance energy transfer-derived structure of a quantum dot-protein bioconjugate nanoassembly" Proc. Natl. Acad. Sci. USA, vol. 101, No. 26, 9612-9617 (2004).

P. T. Snee, R. C. Somers, G.Nair, J. P. Zimmer, M. G. Bawendi, D. G. Nocera. "A Ratiometric CdSe/ZnS Nanocrystal pH Sensor" J. Am. Chem. Soc., vol. 128, 13320-13321 (2006).

Balzani V., Ceroni P., Gestermann S.; Gorka M., Kauffmann C. and Vogtle F., "Fluorescent guests hosted in fluorescent dendrimers" Tetrahedron, vol. 58, 629-637 (2002).

Jares-Erijman, E.A., and Jovin,T.M., "Reflections on FRET imaging: formalism, probes, and implementation" In FRET and FLIM Imaging Techniques. T. Gadella Jr., Editor. Wiley chapter 12, 475-517 (2009). Menendez G., Jovin T.M., and Jares-Erijman E.A., in preparation. (2009) 

"Nucleation of protein fibrillation by nanoparticles" Proc. Natl. Acad. Sci. USA, vol. 104, No. 21, 8691-8696 (2007).

[28] Roberti, M.J., Morgan, M., Pietrasanta, L.I., Jovin, T.M., and Jares-Erijman, E.A., “Quantum dots as ultrasensitive nanoactuators and sensors of amyloid aggregation in live cells", 2009, submitted.

[29] Chandra, S. , Gallardo, G., Fernández-Chacón, R., Schlüter, O. and Südhof, T." $\alpha$-synuclein cooperates with CSP $\alpha$ in preventing neurodegeneration" Cell, vol. 123, No. 3, 2005, pp. 383-96.

[30] Roberti, M.J., Bertoncini, C., Klement, R., Jares-Erijman, E.A., Jovin, T.M, "Fluorescence imaging of amyloid formation in living cells by a functional, tetracysteine-tagged $\alpha$-synuclein" Nat. Methods, vol. 4, No. 4, 345351 (2007).

[31] Spagnuolo, C., Vermeij, R., Jares-Erijman, E.A., "Improved photostable FRET-competent biarsenicaltetracysteine probes based on fluorinated fluoresceins" J. Am. Chem. Soc., vol. 128, No. 37, 12040-12041 (2006). 